\title{
Identification of Ellis rheological law from free surface velocity
}

\author{
Abdulrahman Al-Behadili ${ }^{\mathrm{a}}$, Mathieu Sellier ${ }^{\mathrm{a}, *}$, James N. Hewett $^{\mathrm{a}}$, Roger I. Nokes $^{\mathrm{b}}$, Miguel Moyers-Gonzalez $^{\mathrm{c}}$ \\ ${ }^{a}$ Department of Mechanical Engineering, University of Canterbury, Christchurch 8140, New Zealand \\ ${ }^{b}$ Department of Civil and Natural Resources Engineering, University of Canterbury, Christchurch 8140, New Zealand \\ ${ }^{c}$ Department of Mathematics and Statistics, University of Canterbury, Christchurch 8140, New Zealand
}

\begin{abstract}
This study presents a new rheometry technique which requires a free surface velocity field as an input. By minimising the difference between observed and simulated data, we show here that it is possible to estimate the three parameters of an assumed Ellis rheological law. The dam-break problem is considered here with molasses as the working fluid. The free surface velocity is evaluated by seeding the free surface with buoyant particles and using particle tracking velocimetry. The parameter identification is successfully tested with "synthetic" data produced by the numerical model. The parameter identification algorithm is shown to be robust even when significant noise is added to the synthetic dataset. For true experimental data, the reconstructed flow curve is within $25 \%$ of the actual one, demonstrating the potential of the method for circumstances where standard rheometry does not apply.
\end{abstract}

Keywords: Lubrication approximation, Ellis model, Parameter identification, Non-Newtonian fluid, Free surface velocity

\section{Introduction}

There are many instances for which a standard rheometer cannot be used to measure the rheology of a fluid. The fluid ${ }^{20}$ may, for example, be too hot, too dangerous, or in too small 5 quantity Sellier (2016). An alternative way to measure the rheology is therefore required in such circumstances. The idea we pursue in the present work is that in a flow bounded by a free surface, perturbations induced by boundary or initial conditions will be transferred to the free surface. Since the corresponding transfer function is expected to be dependent on the fluid rheology, it is expected that the free surface velocity field contains information about the rheology and we can therefore use this as a proxy to extract information about the fluid rheology. Sellier (2016) reviewed earlier studies describing methods to infer 15 the rheology of a fluid from free surface data. In a recent contribution, we have shown that it is possible to identify the two parameters of a power-law rheology by minimising the differ-

\footnotetext{
${ }^{*}$ Corresponding author

Email address: mathieu.sellier@canterbury.ac.nz (Mathieu
} Sellier) ence between the measured and simulated free surface velocity field for the dam-break flow problem Al-Behadili et al. (2018). The power-law rheology is however quite restrictive and is unable to describe the behaviour of many fluids, particularly for low shear values Myers (2005). In this paper, we therefore aim to demonstrate that it is possible to extend the methodology described in Al-Behadili et al. (2018) to identify the parameters of an Ellis rheological law which is more versatile since it is described by three parameters. The identification process first requires a good model of free surface flows of an Ellis fluid. The modelling of the free surface flow of an Ellis fluid has been the topic of several studies. Gravity-driven flow down an inclined plane was investigated for plane flow in Weidner and Schwartz (1994); Myers (2005) and for three-dimensional flow in Schwartz and Eley (2002); Kheyfets and Kieweg (2013). Coating applications have motivated several studies involving the free surface flow of an Ellis fluid including spin coating 35 Charpin et al. (2007), dip coating or the related Landau-Levich problem for a plane surface Tallmadge (1966); Afanasiev et al. (2007); Hewson et al. (2009) or a cylinder Roy (1971). Ellis 
rheology was also used to describe the dynamics of a tear film on the eye Jossic et al. (2009); Braun et al. (2012). The model using a grid search.

\section{Description of the experimental procedure}

\subsection{Experimental setup}

The experiment was designed to obtain the free surface velocity for the dam-break problem. A tank was constructed from Perspex and was divided by a removable gate as depicted in Figure 1. Images of the free surface were captured with a $1280 \times 1024$ px, 30 frames per second, Motion Pro X $3_{100}$

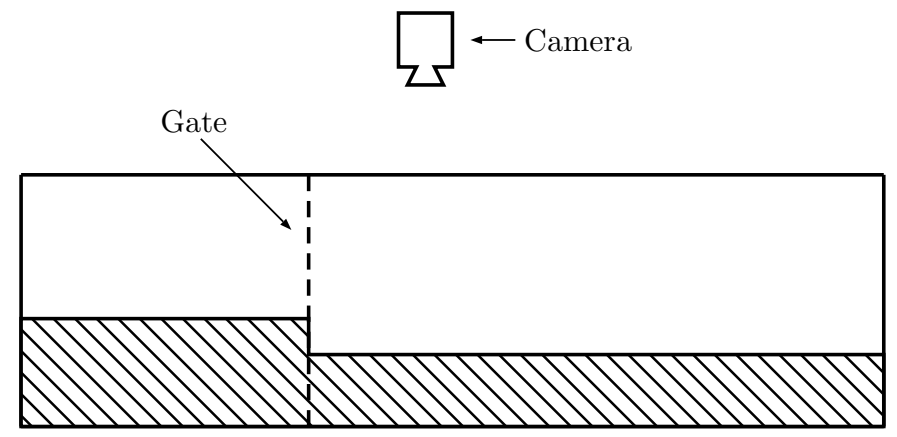

Figure 1: Side view of the experimental setup for the dam-break problem, where the filled in hatched area represents the initial condition of the fluid.

height speed camera with a $55 \mathrm{~mm}$ Nikon lens attached. The camera was located directly above of the tank, at a height of $1.8 \mathrm{~m}$ perpendicular to the free surface. The spatial resolution recorded by the camera was $0.353 \mathrm{~mm} / \mathrm{px}$. Four fluorescent lights (Phillips 58W/865) were used as a lighting system and placed around two sides of the tank (with two tubes on each side). White Acrylic sheets with a thickness of $3 \mathrm{~mm}$, were placed along two sides of the tank in order to diffuse the light and obtain a uniform lighting intensity. The working fluid was a sugar cane molasses. Initially there was a $12 \mathrm{~mm}$ difference in fluid depth on either side of the gate, with a depth of $20 \mathrm{~mm}$ on the upstream side and $8 \mathrm{~mm}$ on the downstream side as shown in Figure 4. The fluid surface on each side of the gate was seeded with buoyant, evenly distributed polystyrene white beads with a diameter of $1 \mathrm{~mm}$. The experiment was initiated by the removal of the gate after the fluid pools on both sides of the gate were observed to be stationary.

\subsection{Particle tracking velocimetry}

PTV was used to calculate the free surface velocity from the images captured by the high speed camera. PTV analysis is a process in which individual particles are tracked within a fluid, and the calculations were performed using the software package Streams (Nokes, 2014). PTV includes four steps: image capture and processing, particle identification, particle matching between frames, and velocity field generation. In Streams, the image sequence represents the starting point of the image processing. The image sequence includes a series of images. 


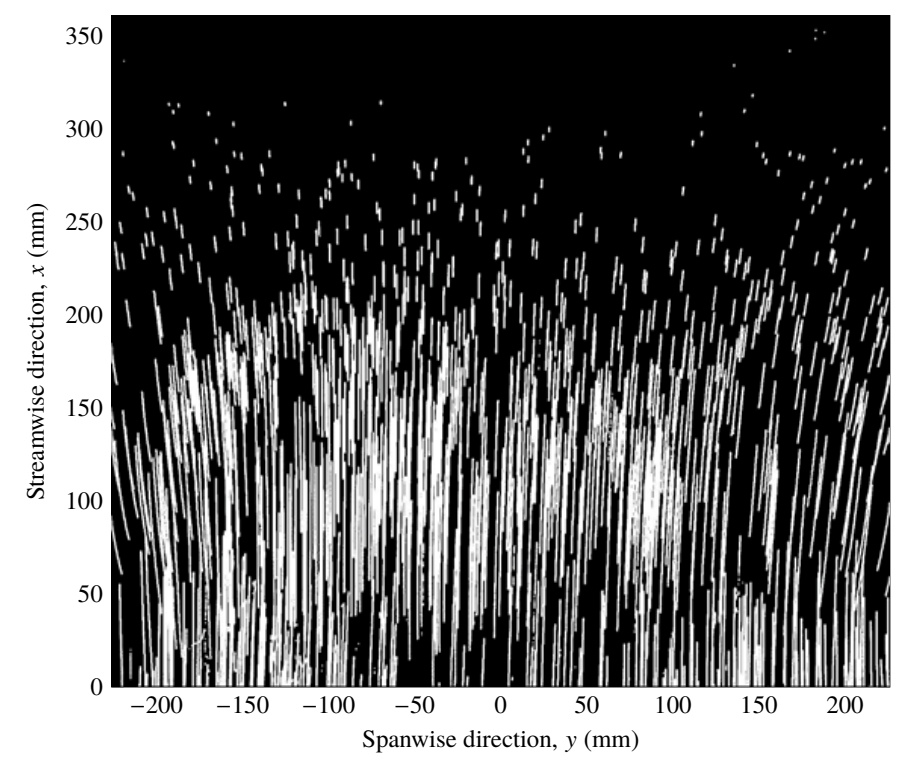

125

Figure 2: Pathlines of the particles at the free surface for molasses from $t=0.0 \mathrm{~s}$ to $t=27.7 \mathrm{~s}$, directly retrieved from the Streams software.

A total of 2500 frames were captured at 30 frames per second in the experiment, and this image sequence was downsampled by a factor of four; yielding a time step of $0.16 \mathrm{~s}$ between frames. The full set of images were downsampled because the molasses flows very slowly due to its high viscosity. The selected time step allows the particles to be further apart between frames and therefore allows for a more accurate description of the flow field. Next, the particle identification process is used to identify particles within each frame to obtain a list of particles with their size, shape, intensity, and location. The critical step within a PTV analysis is the tracking of particles between ${ }_{135}$ frames, since this allows calculation of their Lagrangian velocity. Streams uses an optimisation algorithm to match particles from one frame to the next. This algorithm identifies correct matches by minimising a cost associated with every potential particle pairs. Once particles have been tracked between frames ${ }_{140}$ and their Lagrangian velocities calculated, the final step within the PTV analysis is to interpolate these velocities onto a regular grid, providing the Eulerian velocity field for the flow. The particle trajectories computed by the PTV algortihm are illustrated in Figure 2. More details on the methodology and its applica-145 tion can be found in Nokes (2014); Campagnol et al. (2013); ?

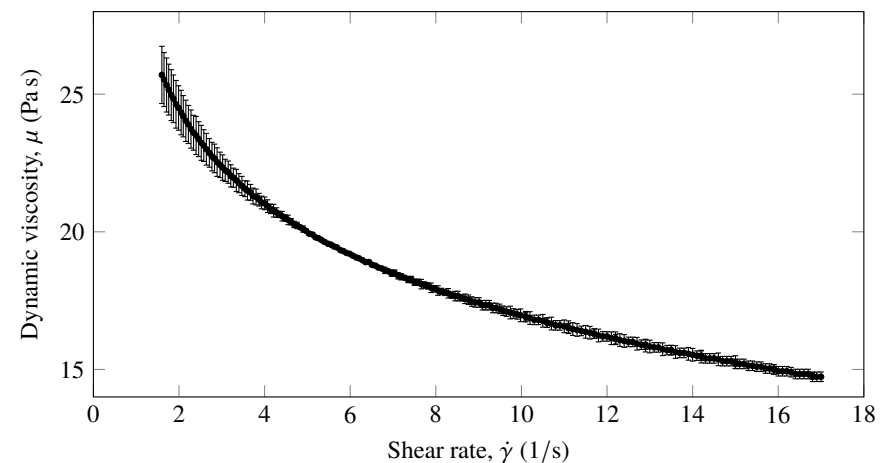

Figure 3: Viscosity of molasses used in the experimentals, as measured by the Rheometer MCR 301

\subsection{Fluid properties}

The rheology of the molasses was measured using a Rheometer MCR 301. Figure 3 illustrates the mean and standard deviation of three tests for the viscosity of molasses at room temperature $\left(20^{\circ} \mathrm{C}\right)$ plotted against the shear rate. The molasses behaved as a non-Newtonian fluid within the shear rate range of interest.

The mathematical expression of the Ellis model is

$\frac{1}{\mu}=\frac{1}{\mu_{0}}\left(1+\left|\frac{\tau}{\tau_{\frac{1}{2}}}\right|^{\alpha-1}\right)$

where $\mu_{0}$ is the zero shear viscosity, $\tau_{\frac{1}{2}}$ the shear stress at which the zero shear viscosity has been reduced by a factor of one-half and $\alpha$ the shear thinning parameter. The fluid is Newtonian for $\alpha=1$ and non-Newtonian when $\alpha>1$ (Charpin et al., 2007). The fluid viscosity $\mu$ depends on these three adjustable parameters as shown in Equation 1, and $\mu$ is dependent on one variable, the fluid shear stress such that $\mu=f(\tau)$. This particular function can be found experimentally from the rheometer data (Schwartz and Eley, 2002). In order to obtain the Ellis rheological parameters of molasses from the rheometer data (Figure 3), we developed a MATLAB code which identifies the optimal Ellis parameters that best matched the rheometer measurements. This code solved the non-linear least square problem to fit the rheometer data by varying the three Ellis parameters. The result from the data fitting code was $\alpha=1.6, \tau_{\frac{1}{2}}=271 \mathrm{~Pa}$ and $\mu_{0}=37.4 \mathrm{~Pa} \mathrm{~s}$.

Similarly, the Carreau rheological parameters of the molasses 
was also obtained using the MATLAB data fitting code, applied to the rheometer data. The mathematical expression of the Carreau model which was used in this data fitting code was from Johnston et al. (2004) and defines the dynamic viscosity as

$\mu=\mu_{\infty}+\left(\mu_{0}-\mu_{\infty}\right)\left(1+\lambda^{2} \dot{\gamma}^{2}\right)^{\left(n_{c}-1\right) / 2}$

where $\mu_{\infty}$ and $\mu_{0}$ are the limiting viscosities at low and high shear rates respectively, $\lambda$ is the characteristic time, $\dot{\gamma}$ is the shear rate and $n_{c}$ is the flow behaviour index of the Carreau model. After implementing the data fitting code with $\mu_{\infty}=$ 0 Pa s (Myers, 2005), it was found that $\mu_{0}=37.4 \mathrm{~Pa} \mathrm{~s}, \lambda=2.94$ and $n_{c}=0.76$ for the molasses. The fluid surface tension of the molasses was taken to be $0.05 \mathrm{~Pa} \mathrm{~m}$ and the density $1450 \mathrm{~kg} / \mathrm{m}^{3}$, (Miller and Pike, 1993).

\section{Description of the mathematical models and numerical} methods

The Navier-Stokes equations describe the full flow behaviour by using the conservation of mass and momentum, with the fluid rheology described in terms of the Ellis model. The lubri-180 cation approximation model is a simplification of the NavierStokes, which assumes negligible inertia, a small aspect ratio and slope of the flow. Consequently, the outcomes from the Navier-Stokes model are considered as the benchmark for validating the lubrication approximation model.

\subsection{Model based on the Navier-Stokes equations}

The two-dimensional Navier-Stokes equations for an incompressible fluid can be defined as

$\rho\left(\frac{\partial u}{\partial t}+u \frac{\partial u}{\partial x}+w \frac{\partial u}{\partial z}\right)=-\frac{\partial p}{\partial x}+\frac{\partial}{\partial x}\left(\mu \frac{\partial u}{\partial x}\right)+\frac{\partial}{\partial z}\left(\mu \frac{\partial u}{\partial z}\right)$ $\rho\left(\frac{\partial w}{\partial t}+u \frac{\partial w}{\partial x}+w \frac{\partial w}{\partial z}\right)=-\frac{\partial p}{\partial z}+\frac{\partial}{\partial x}\left(\mu \frac{\partial w}{\partial x}\right)+\frac{\partial}{\partial z}\left(\mu \frac{\partial w}{\partial z}\right)+\rho g$

$\frac{\partial u}{\partial x}+\frac{\partial w}{\partial z}=0$

where $\rho$ is the fluid density, $\mu$ the dynamic viscosity of the fluid, $g=-9.81 \mathrm{~m} / \mathrm{s}^{2}$ the gravitational acceleration and $u$ and $w$ are the fluid velocities in the $x$ and $z$ directions respectively. The

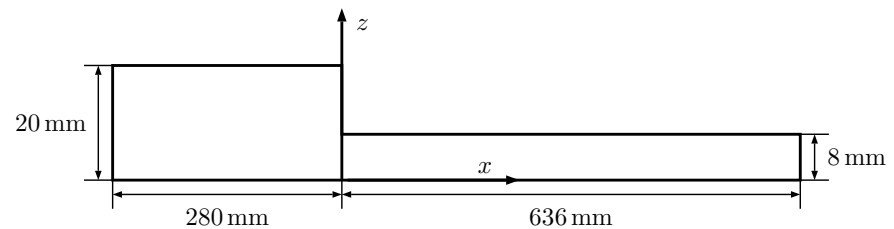

Figure 4: Coordinate system with an aspect ratio of 0.015 (the aspect ratio is defined as the ratio of the average fluid height and the horizontal length of the domain).

Navier-Stokes equations describes the motion of fluid and can be used to model either Newtonian or non-Newtonian fluids, depending on which rheological law for $\mu$ is employed. The relative importance of the various forces at play during the flow is estimated using dimensionless numbers. The relevant ones for the dam-break flow of interest here are the Reynolds number $R e=\frac{\rho U_{0} H_{0}}{\mu}$ (ratio of inertia to viscous forces), the Froude number $F r=\frac{U_{0}}{\sqrt{g H_{0}}}$ (ratio of inertia to gravity forces), the capillary number $C a=\frac{\mu U_{0}}{\sigma}$ (ratio of viscous to surface tension forces) and the Weber number $W e=\frac{\rho H_{0} U_{0}^{2}}{\sigma}$ where $\sigma$ is the surface tension of the fluid (ratio of inertia to surface tension forces). The average film thickness $H_{0}$ and the characteristic velocity $U_{0}$ are used in the definition of the dimensionless numbers. The Navier-Stokes equations were solved using the finite element software, COMSOL Multiphysics 5.0. The two-dimensional, transient, laminar two-phase flow model with the moving mesh interface of COMSOL was used to simulate the traversing fluid in this dam-break problem. The full incompressible NavierStokes equations were solved in a domain which was deformed by the moving free surface using the Arbitrary LagrangianEulerian (ALE) method. The ALE allows the mesh to conform to the evolving fluid domain as the free surface transforms over time. The Winslow mesh smoothing technique was used for propagating the interface displacement throughout the domain. The initial geometry for the computational domain was composed of two adjacent rectangles (representing the fluid pools on either side of the gate), with dimensions as shown in Figure 4. The initial free surface of the fluid was composed of the top edges of the domain, and the sharp corners at $x=0 \mathrm{~mm}$ were smoothed with $2 \mathrm{~mm}$ fillets, in order to avoid remeshing in 
the solution. The domain was discretised with an unstructured

\subsection{Model based on the lubrication approximation}

The shear stress of the fluid $\tau$ is defined as

$\tau=\mu \dot{\gamma} \quad$ with $\quad \dot{\gamma}=\frac{d u}{d z}$

where $\dot{\gamma}$ is the shear rate of the fluid. The viscosity is a function of the shear stress (Schwartz and Eley, 2002), such that

$\mu=f(\tau)$

Considering an infinitesimal volume element within the fluid, the sum of the forces is equal to zero since the fluid does not undergo acceleration in the lubrication approximation case; due to the negligible inertia assumption. The force balance leads to the following mathematical expression (Schwartz and Eley. 2002)

$\frac{d \tau}{d z}=-\frac{d p}{d x} \Longrightarrow \tau=\left(-\frac{d p}{d x}\right) z$

Then from Equation 6 and 7 the shear rate can be expressed as

$\frac{d u}{d z}=\frac{\tau}{f(\tau)}$

It is clear that from Equation $8, f(\tau)=f(z)$, and this relation leads to

$\frac{d u}{d z}=\frac{\tau}{f(z)}$

Substitution $\mu=f(\tau)=f(z)$ and $\tau=\left(-\frac{d p}{d x}\right) z$ into Equation 1 yields

$\frac{1}{f(z)}=\frac{1}{\mu_{0}}\left(1+\left|\frac{\left(-\frac{d p}{d x}\right) z}{\tau_{\frac{1}{2}}^{\alpha-1}}\right|^{\alpha}\right)$
Substituting Equation 11 into Equation 10 and integrating with respect to $z$ from the substrate $(z=0)$ to the free surface $(z=h)$, yields an expression for the horizontal free surface velocity $u_{s}$ which is based on the Ellis model (Kheyfets and Kieweg, 2013) such that

$u_{s}=\frac{1}{\mu_{0}}\left(\frac{\left(-\frac{d p}{d x}\right) h^{2}}{2}+\left(-\frac{d p}{d x}\right) \frac{h^{\alpha+1}}{\alpha+1}\left|\frac{\left(-\frac{d p}{d x}\right)}{\tau_{\frac{1}{2}}}\right|^{\alpha-1}\right)$

The corresponding vertical free surface velocity will be

$w_{s}=\frac{\partial h}{\partial t}+u_{s} \frac{\partial h}{\partial x}$

Conservation of mass requires that

$\frac{\partial h}{\partial t}+\frac{\partial Q}{\partial x}=0, \quad Q=\int_{0}^{h} u d z$

The velocity and flux expressions (Equations 12 and 14 ) are incorporated into the momentum and conservation equations to obtain a time-dependent partial differential equation, which describes the free film thickness $h$ with

$\frac{\partial h}{\partial t}+\frac{\partial}{\partial x}\left(\frac{-p_{x}}{\mu_{0}}\left(\frac{h^{3}}{3}+\frac{h^{\alpha+2}}{\alpha+2}\left(\frac{\left|p_{x}\right|}{\tau_{\frac{1}{2}}}\right)^{\alpha-1}\right)\right)=0$

For low Reynolds number and unidirectional flow with small aspect ratios, Equation 4 reduces to

$\frac{\partial p}{\partial z}=\rho g \Longrightarrow p(x, z, t)=\rho g z+f(x, t)$

The boundary condition of the pressure at the free surface is

$p(x, z, t)=-\sigma \frac{\partial^{2} h}{\partial x^{2}}$

Integrating Equation 16 with respect to $z$, subject to boundary condition Equation 17, yields

$$
\begin{aligned}
& p(x, z=h, t)=\rho g h+f(x, t)=-\sigma \frac{\partial^{2} h}{\partial x^{2}} \\
& \Longrightarrow f(x, t)=-\sigma \frac{\partial^{2} h}{\partial x^{2}}-\rho g h \\
& \Longrightarrow p(x, z, t)=-\rho g(h-z)-\sigma \frac{\partial^{2} h}{\partial x^{2}}
\end{aligned}
$$

Equations 15 and 20 represent the lubrication approximation based on the Ellis model which is an approximation of the Navier-Stokes equations.

The equations in the lubrication approximation model were also solved numerically with COMSOL. A smoothed step function was used to describe the initial free surface level for the 
dam-break problem. The first step was to calculate the free surface level $h(x, t)$ by solving Equation 15, and then the free surface velocity was computed using Equation 12 In order to solve the lubrication approximation equations in COMSOL, Equations 15 and 20 were rewritten in a standard form for the solver, with the coefficients matching

$e_{a} \frac{\partial^{2} u}{\partial t^{2}}+d_{a} \frac{\partial u}{\partial t}+\nabla \cdot(-c \nabla u-\alpha u+\gamma)+\beta \cdot \nabla u+a u=f, \quad u=[h, p]^{T}$

where the solution was based on two dependent variables $h$ and $p$. A zero flux boundary condition was applied at both ends of the computational domain, with

$\frac{\partial h}{\partial x}=0 \quad$ and $\quad \frac{\partial p}{\partial x}=0$

\subsection{Verification of the lubrication approximation model}

The time-dependent lubrication approximation equations based on the Ellis model (Equations 15 and 20) were validated against the Navier-Stokes solution of the dam-break problem. The domain was divided into 200 quadratic elements (onedimensional), with a total length of $916 \mathrm{~mm}$ for the lubrication approximation model. For this verification case, the nonNewtonian fluid employed was Polyethylene oxide; with the Ellis rheological model parameters $\alpha=3, \tau_{\frac{1}{2}}=20 \mathrm{~Pa}$ and $^{240}$ $\mu_{0}=15.25 \mathrm{Pas}$, and for the Carreau model $\mu_{0}=15.25 \mathrm{Pas}$, $\lambda=1.18 \mathrm{~s}$ and $n_{c}=0.41$ (Myers, 2005). In the simulations, the surface tension $\sigma$ is taken to be $0.05 \mathrm{Pam}$ and the density $\rho=1450 \mathrm{~kg} / \mathrm{m}^{3}$.

Figure 5 shows the comparison between the solutions obtained for the lubrication approximation and the full NavierStokes equations at three different times; namely $0.5 \mathrm{~s}, 5 \mathrm{~s}$ and $10 \mathrm{~s}$. The agreement between the lubrication approximation and the Navier-Stokes for the film thickness over time is good.245 However, there is a small discrepancy between the models because the lubrication approximation contains several assumptions, yielding an approximation to the Navier-Stokes equations. These assumptions are that the inertia terms are neglected and that the aspect ratio of the fluid layer must be sufficiently 250

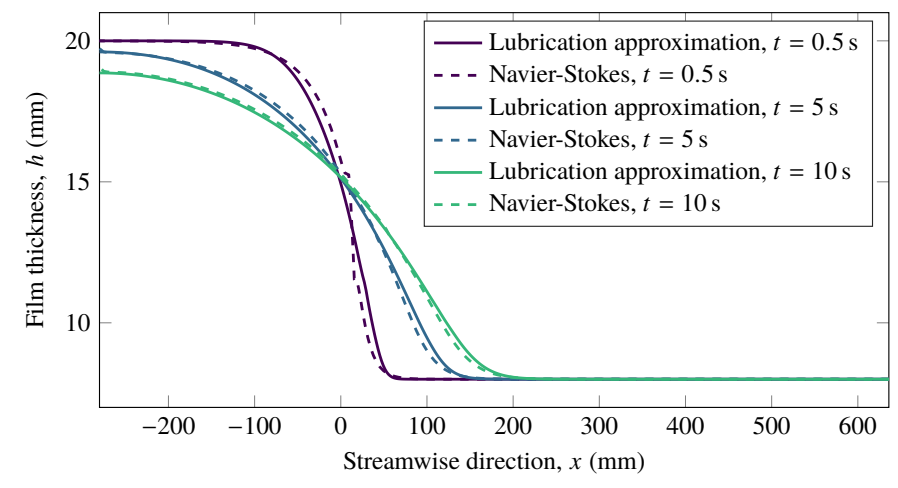

Figure 5: Polyethylene oxide film thickness along the tank over time for two different numerical models (Lubrication approximation and Navier-Stokes).

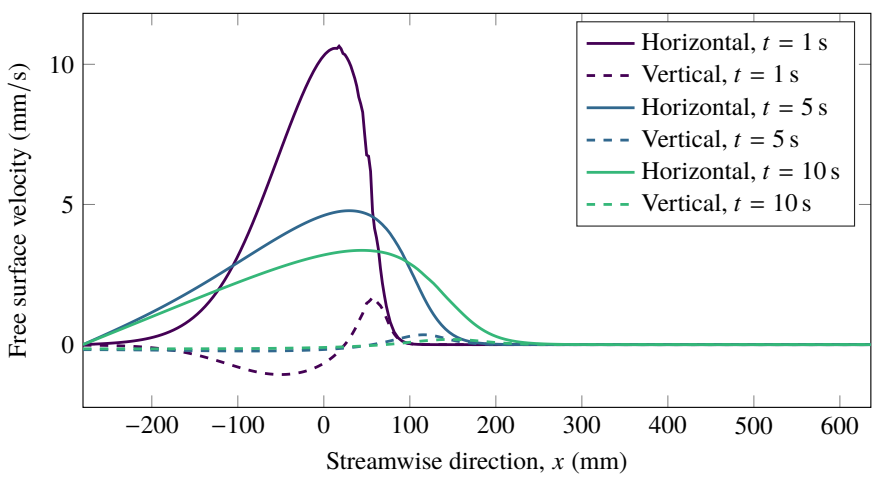

Figure 6: Horizontal and vertical velocity components for the dam-break flow of Polyethylene oxide.

small. A similar comparison between the lubrication approximation and full Navier-Stokes models was performed in (Morris et al., 2017) for Newtonian fluids.

The absolute error percentage $\delta$ between the two models was calculated as

$\delta=\left(\frac{1}{L} \int_{0}^{L} \frac{\left|h(x, t)_{\mathrm{LA}}-h(x, t)_{\mathrm{NS}}\right|}{h(x, t)_{\mathrm{NS}}} d x\right) \%$

where $h(x, t)_{\mathrm{LA}}$ is the film thickness for the lubrication approximation and $h(x, t)_{\mathrm{NS}}$ for the Navier-Stokes model. The absolute error percentage at $0.5 \mathrm{~s}, 5 \mathrm{~s}$ and $10 \mathrm{~s}$ time is $0.75 \%, 0.51 \%$ and $0.42 \%$ respectively. These errors reduce over the duration of the simulation because the effect from the different initial conditions diminishes over time; a step function at the gate was applied with smoothing for both the lubrication approximation and the Navier-Stokes models in order to ensure a smooth, continuous free surface height. Figure 6 shows the corresponding horizontal and vertical components of the free surface velocity 
calculated according to Equations 12 and 13 , respectively. This figure confirms that the vertical velocity component is signifi-280 cantly smaller than the horizontal one which is measured and used in the parameter identification algorithm. Using $0.01 \mathrm{~m} / \mathrm{s}$ as the characteristic velocity $U_{0}$, we find the viscosity given by Equation 1 to be $\mu=7.45 \mathrm{~Pa}$ s and the corresponding dimensionless numbers $\operatorname{Re}=2.72 \times 10^{-2}, \mathrm{Fr}=2.70 \times 10^{-2}, 285$ $C a=1.49$, and $W e=4.06 \times 10^{-2}$. These dimensionless numbers confirm that inertia is negligible compared to viscous, gravity, and surface tension forces and viscous and surface tension forces are of equal order of magnitude.

\section{Parameter identification}

\subsection{Identification with synthetic data}

In this section, we first create a synthetic data set for the free surface velocity by numerically solving the lubrication approx- ${ }_{295}$ imation model with prescribed Ellis rheology parameters. A parameter identification technique is then applied on this synthetic data in order to find the optimal values of, and ultimately rediscover, the rheology parameters. Two non-Newtonian fluids were used, namely Polyethylene oxide and Hydroxylethycellulose; their corresponding Ellis parameters are listed in Table 1 . In order to identify the Ellis parameters, a parametric identification study was carried out by performing a grid search. The range of parameters explored in the grid search was $0.1 \leqslant \mu_{0} \leqslant$ $100,0.1 \leqslant \alpha \leqslant 4$ and $1 \leqslant \tau_{\frac{1}{2}} \leqslant 300$. An objective function $F$ was defined for $N$ times and evaluated with the experimental data using

$F\left(\mu_{0}, \tau_{\frac{1}{2}}, \alpha\right)=\sum_{i=1}^{N} 0.5 \int_{0}^{L}\left(u_{c}^{i}(x)-u_{m}^{i}(x)\right)^{2} d x$

where $u_{m}$ is the measured free surface velocity obtained from the experiment and $u_{c}$ the computed velocity from the lubrication approximation (Equation 12). The $F$ was evaluated once; such that $N=1$.

In order to identify the three parameters of the Ellis model using the grid search, we first fixed one of the three parameters by setting it equal to a specific value within the parametric space.
We then implemented a parametric study for the full range of the other two parameters to obtain the corresponding minimum $F$ on this parametric plane. The next step was to select one of the two other parameters and then fix this parameter and repeat the process. This procedure narrowed down the parametric space until the global minimum of $F$ was reached.

The results from the final three iterations of the parametric search is shown in Figure 7 with contour plots, which includes three minimum $F$ for both fluids. The first comparison is between $\mu_{0}$ and $\alpha$, the second is between $\alpha$ and $\tau_{\frac{1}{2}}$ and the third is between $\mu_{0}$ and $\tau_{\frac{1}{2}}$.

The number of mesh elements in this parametric study was 229 and the aspect ratio was 0.0152. A smoothed step function was used to describe the initial free surface level for the dam-break region of the flow. The Ellis rheology parameters corresponding to the global minimum of the $F$ was found (reconstructed case), are listed in Table 1, and agree well with the expected values (actual case).

\subsection{Identification with noisy synthetic data}

The sensitivity of our lubrication approximation model was evaluated by applying noise for the Polyethylene oxide and Hydroxylethycellulose fluids in order to determine the robustness of the parameter identification process. Artificial noise was added to the synthetic data and then the parameter identification process was repeated. The $F$ in Equation 24 can be rewritten to include this noise component with

$F\left(\mu_{0}, \tau_{\frac{1}{2}}, \alpha\right)=\sum_{i=1}^{N} 0.5 \int_{0}^{L}\left(u_{c}^{i}(x)-\left(u_{m}^{i}(x)+\varepsilon\right)\right)^{2} d x$

where the noise is defined as

$\varepsilon=\left(u_{\max }-u_{\min }\right) E r$

where, $u_{\max }$ and $u_{\min }$ represent the maximum and minimum values of the free surface velocity respectively, $r$ the percentage of the added noise and $E$ the random values with $-1 \leqslant E \leqslant 1$.

The rheological parameters were then re-evaluated with a range of percentage of added noise, up to $40 \%$, for both fluids and compared with the synthetic data without noise; results 

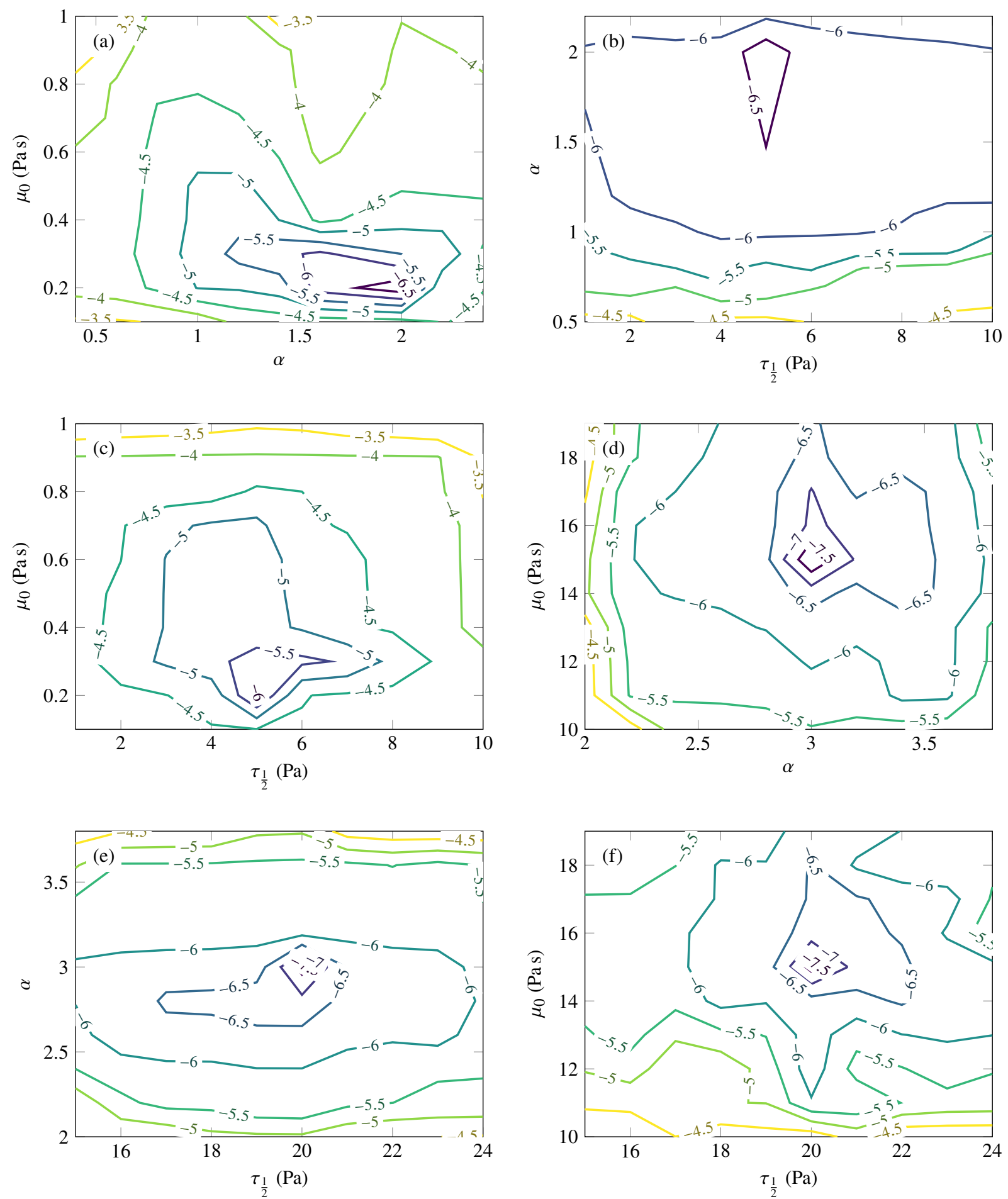

Figure 7: Contour lines for the $\log$ of the objective function, $\log (F)$, with respect to the rheological parameters, near the optimum solution, obtained from the parametric study with synthetic data for two non-Newtonian fluids, namely: (1) Hydroxylethycellulose, (a) $\tau_{\frac{1}{2}}=5 \mathrm{~Pa}$, (b) $\mu_{0}=0.22 \mathrm{Pas}$, (c) $\alpha=2$; and (2) Polyethylene oxide (d) $\tau_{\frac{1}{2}}=20 \mathrm{~Pa}$, (e) $\mu_{0}=15.25 \mathrm{Pas}$ (f) $\alpha=3$. 
Table 1: Actual Myers 2005) and reconstructed values for two non-Newtonian fluids for the verification of the parametric identification process.

\begin{tabular}{llccc}
\hline Fluid & Case & $\tau_{\frac{1}{2}}(\mathrm{~Pa})$ & $\mu_{0}(\mathrm{Pas})$ & $\alpha$ \\
\hline Hydroxylethycellulose & Actual & 5 & 0.22 & 2 \\
& Reconstructed & $5.0 \pm 0.5$ & $0.220 \pm 0.005$ & $2.00 \pm 0.05$ \\
Polyethylene oxide & Actual & 20 & 15.25 & 3 \\
& Reconstructed & $20.0 \pm 0.5$ & $15.25 \pm 0.50$ & $3.00 \pm 0.05$ \\
\hline
\end{tabular}

Table 2: The Ellis model rheological parameters for the synthetic data cases with added noise.

\begin{tabular}{lccccc}
\hline Fluid & Added noise $(\%)$ & $\alpha$ & $\mu_{0}(\mathrm{Pas})$ & $\tau_{\frac{1}{2}}(\mathrm{~Pa})$ & $F_{\min }\left(\mathrm{m}^{3} / \mathrm{s}^{2}\right)$ \\
\hline Hydroxylethycellulose & 0 & $2.00 \pm 0.05$ & $0.220 \pm 0.005$ & $5.0 \pm 0.5$ & $1.53 \times 10^{-7}$ \\
& 2 & $2.00 \pm 0.05$ & $0.230 \pm 0.005$ & $4.0 \pm 0.5$ & $2.36 \times 10^{-7}$ \\
& 3 & $2.10 \pm 0.05$ & $0.250 \pm 0.005$ & $4.0 \pm 0.5$ & $2.83 \times 10^{-7}$ \\
& 4 & $2.10 \pm 0.05$ & $0.230 \pm 0.005$ & $5.0 \pm 0.5$ & $4.17 \times 10^{-7}$ \\
Polyethylene oxide & 10 & $2.10 \pm 0.05$ & $0.230 \pm 0.005$ & $5.0 \pm 0.5$ & $8.71 \times 10^{-7}$ \\
& 20 & $2.10 \pm 0.05$ & $0.230 \pm 0.005$ & $5.0 \pm 0.5$ & $6.20 \times 10^{-6}$ \\
& 40 & $2.00 \pm 0.05$ & $0.270 \pm 0.005$ & $5.0 \pm 0.5$ & $2.56 \times 10^{-5}$ \\
& 0 & $2.70 \pm 0.05$ & $15.25 \pm 0.50$ & $20.0 \pm 2.5$ & $2.05 \times 10^{-8}$ \\
& 2 & $2.50 \pm 0.05$ & $14.25 \pm 0.50$ & $25.0 \pm 2.5$ & $2.31 \times 10^{-8}$ \\
& 3 & $2.50 \pm 0.05$ & $14.25 \pm 0.50$ & $25.0 \pm 2.5$ & $2.49 \times 10^{-8}$ \\
& 4 & $2.50 \pm 0.05$ & $14.25 \pm 0.50$ & $25.0 \pm 2.5$ & $3.45 \times 10^{-8}$ \\
& 10 & $2.50 \pm 0.05$ & $14.25 \pm 0.50$ & $25.0 \pm 2.5$ & $1.50 \times 10^{-7}$ \\
& 20 & $2.40 \pm 0.05$ & $14.25 \pm 0.50$ & $20.0 \pm 2.5$ & $5.50 \times 10^{-7}$ \\
& 40 & $2.70 \pm 0.05$ & $12.25 \pm 0.50$ & $45.0 \pm 2.5$ & $2.18 \times 10^{-6}$ \\
\hline
\end{tabular}

are shown in Table 2. The values of the minimum $F$ are slightly

\subsection{Identification with experimental data}

We experimentally analysed the dam-break classical flow with molasses as described in Section 2 Figure 8 shows the variation of the free surface velocity downstream of the gate at the flow reduces from a peak value at $x=0 \mathrm{~mm}$. This peak velocity decreases over time and the velocity decays with distance from the gate. This drop in velocity was caused by the highly viscous fluid. Given an estimated characteristic velocity $U_{0} \sim 0.01 \mathrm{~m} / \mathrm{s}$, the corresponding dimensionless numbers are $R e=6.66 \times 10^{-3}, F r=2.70 \times 10^{-2}, C a=6.1$, and $W e=4.06 \times 10^{-2}$. These dimensionless numbers show, as before, that inertia is negligible compared to viscous, gravity, and surface tension forces and viscous and surface tension forces are equally important.

Free surface velocity data was used for the identification process based on the Ellis model, and we found for the mo- 


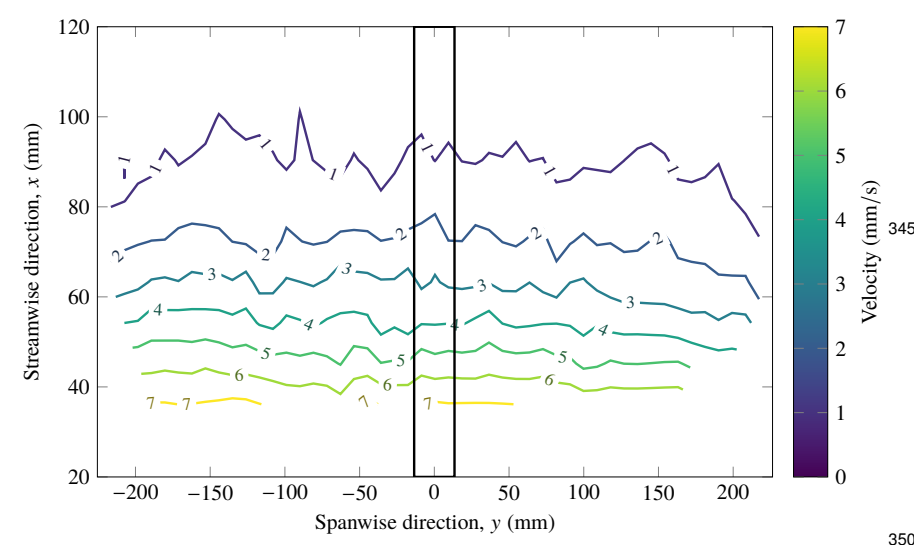

Figure 8: Close-up snapshot of the free surface velocity magnitude for the dambreak problem using molasses as the fluid, shortly after releasing the gate, at time $t=1.33 \mathrm{~s}$. The lines at the centre outline the region where values are obtained for averaging the velocity field data.

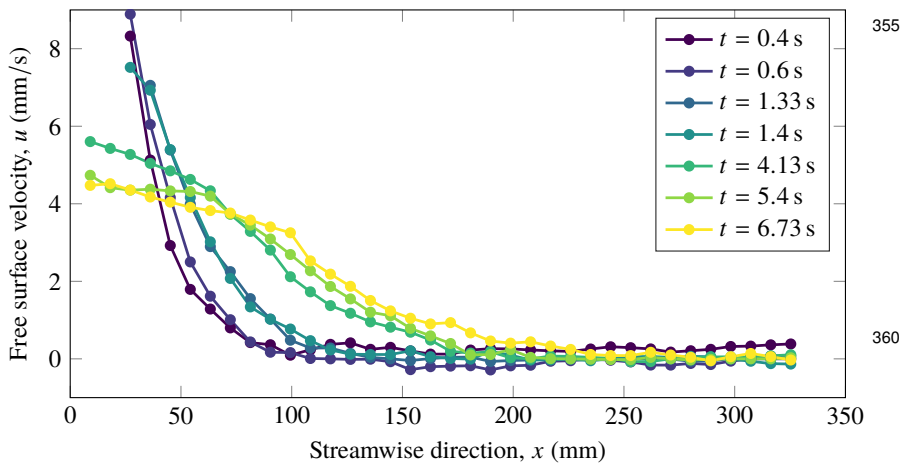

Figure 9: Evolution of the free surface velocity for the dam-break experiment using molasses as the fluid.

lasses that $\mu_{0}=(34.0 \pm 0.5) \mathrm{Pa} \mathrm{s}, \alpha=1.10 \pm 0.05$ and $\tau_{\frac{1}{2}}={ }_{365}$ $(290.0 \pm 2.5) \mathrm{Pa}$. A summary of the results is shown in Table 3 where a subset of the results near the final solution is listed.

The transient velocity field based on the Ellis model was simulated by applying the rheological parameters obtained from the parametric study into Equation 1. Similarly, the velocity 370 field based on the rheometer data was simulated by applying the rheological parameters determined for molasses into Equation 1 Figure 10 shows the velocity profiles for several instances of time after the gate has been released, and the agreement between the experimental, Ellis model and rheometer for 375 the seven velocity profiles is reasonably good. The PTV of the experiments were not able to capture the peak velocities at $0.4 \mathrm{~s}$ and $0.6 \mathrm{~s}$. There are some differences between the experimen- tal and simulated results due to the experimental uncertainties and the assumptions made for the lubrication approximation model. A single camera can only capture the velocity in twodimensional space, such that the out-of-plane velocity component was unresolved, and therefore the vertical movement of waves were not measured. For the cases considered here, the contribution from the vertical component of velocity was negligible, see Figure 6. The measurement of the fluid level had an accuracy of $0.1 \mathrm{~mm}$. Furthermore, the measurement of the horizontal level of the test rig was measured with a spirit level, which had an accuracy of $1 \mathrm{~mm}$ per $1 \mathrm{~m}$. In addition, the initial time of the experiment $(t=0 \mathrm{~s})$ is an important factor because the timeline of the experiment must coincide with that of the simulation. The time between photographs for the experiment was small $(0.03 \mathrm{~s})$, which means that the action of pulling the gate would affect the results; such as the speed of removing the gate. If determining the time of removing the gate was inaccurate, for example by $0.1 \mathrm{~s}$, then the timing would be three frames out. The main assumptions of the lubrication model are: neglecting the inertia term from the Navier-Stokes equations, and using a small aspect ratio and slope.

\subsection{Navier-Stokes solution}

The Navier-Stokes solver in COMSOL Multiphysics 5.0 has two inbuilt rheological models: the power law model and the Carreau model. The Carreau model outcomes are identical with the Ellis model in terms of the film thickness, velocity and viscosity profiles (Myers, 2005). Consequently, the Navier-Stokes solver which is based on the Carreau model was used in the comparison with the lubrication approximation solver based on the Ellis model. After obtaining the Carreau rheological parameters from the data fitting code (Section 2.3), these parameters were used in COMSOL with a numerical setup similar to that described in Section 3

The Ellis rheological parameters of molasses obtained from the rheometer data fitting solution were used to solve the lubrication approximation equation in COMSOL. Firstly, the two finite element models were solved in order to obtain two film 

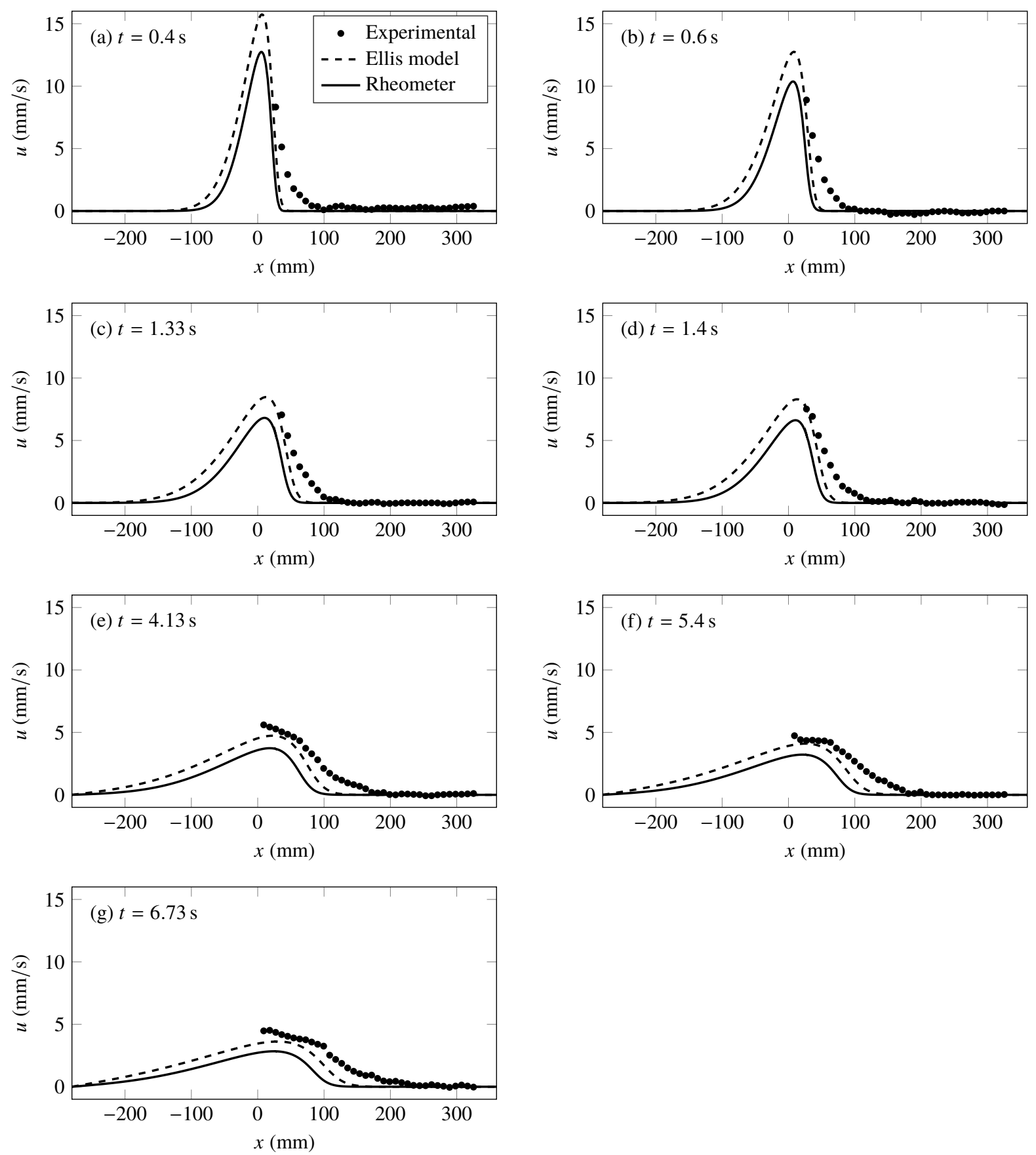

Figure 10: Comparison between the experimental data and the computed velocity based on the Ellis model using rheological parameters determined from the experiment and the rheometer data. 
Table 3: The Ellis model rheological parameters obtained from experimental data of molasses.

\begin{tabular}{ccccccccc}
\hline Stage & Constraint & \multicolumn{4}{c}{ Parameter space } & \multicolumn{4}{c}{ Optimum solution } \\
& & $\alpha$ & $\mu_{0}(\mathrm{~Pa} \mathrm{~s})$ & $\tau_{\frac{1}{2}}(\mathrm{~Pa})$ & $F\left(\mathrm{~m}^{3} / \mathrm{s}^{2}\right)$ & $\alpha$ & $\mu_{0}(\mathrm{~Pa} \mathrm{~s})$ & $\tau_{\frac{1}{2}}(\mathrm{~Pa})$ \\
\hline 1 & $\alpha$ & 1.2 & $30,35, \ldots 75$ & $210,220, \ldots, 300$ & $3.940 \times 10^{-9}$ & 1.2 & 35 & 290 \\
2 & $\mu_{0}$ & $0.8,0.9, \ldots, 1.7$ & 35 & $210,220, \ldots, 300$ & $3.776 \times 10^{-9}$ & 1.1 & 35 & 290 \\
3 & $\tau_{\frac{1}{2}}$ & $0.8,0.9, \ldots, 1.7$ & $30,35, \ldots 75$ & 290 & $3.776 \times 10^{-9}$ & 1.1 & 35 & 290 \\
4 & $\alpha$ & 1.1 & $30,31, \ldots, 39$ & $285,290, \ldots, 330$ & $3.586 \times 10^{-9}$ & 1.1 & 34 & 290 \\
5 & $\mu_{0}$ & $0.9,1.0, \ldots, 1.3$ & 34 & $285,290, \ldots, 330$ & $3.586 \times 10^{-9}$ & 1.1 & 34 & 290 \\
6 & $\tau_{\frac{1}{2}}$ & $0.9,1.0, \ldots, 1.3$ & $30,31, \ldots, 39$ & 290 & $3.586 \times 10^{-9}$ & 1.1 & 34 & 290 \\
\hline
\end{tabular}

thickness profiles. Secondly, we used the free surface velocity

expressions to obtain two velocity profiles.

In Figure 11, it is clear that the agreement in both cases is good. The above comparison process was done by using the velocity profiles and the film thickness. Another comparison ${ }^{410}$ process was implemented by using the shear rate-viscosity profiles. Two data sets were used, namely the rheometer data (Section 2.3 and the parametric identification data based on the Ellis model (Section 4.2). The rheometer profile was plotted by applying Equation 1 The three rheological parameters in $^{415}$ Equation 1 was obtained from the Ellis MATLAB code. Similarly, the parametric identification profile was obtained from using Equation 1 with the Ellis rheological parameters which were obtained from the parametric study. The outcome of the above mentioned comparison process is shown in the Figure 12. This figure shows the comparison between the rheometer ${ }_{420}$ ological parameters in Equation 1 were obtained from the Ellis data fitting code. Similarly, the parametric identification profile was obtained from using Equation 11 with the Ellis rheological parameters obtained from the parametric study. The recon- 425 structed and actual flow curves are shown in Figure 12. There clearly are differences between the two curves especially at low shear rates where the maximum difference between actual and reconstructed viscosity is around $25 \%$. For higher shear rates, the agreement between the two flow curves improves. The dif- 430 ference between the two curves may be explained by a num- ber of factors. Firstly, generating a reliable free surface velocity proved challenging with the experimental set-up. The bead sparsity made the velocity field under-resolved and noiseto-signal ratio increased significantly when the flow velocity decreased substantially degrading the quality of the data. Secondly, the flow experiences a limited range of shear rates as illustrated in Figure 13 where the peak value at $t=0.4 \mathrm{~s}$ is $\dot{\gamma}_{\max }=5.2 \mathrm{~s}^{-1}$. Intuitively, it is therefore clear that the method cannot possibly probe rheological parameters outside of this shear rate range. Moreover, the noise-to-signal ratio is highest when the velocity is smallest which corresponds to the smallest shear rates. Hence, we would expect the reconstructed rheology to be weakest for the lowest values of the shear rate.

\section{Conclusion}

The free surface velocity for a classical flow, the dam-break problem, was measured experimentally using particle tracking velocimetry. A mathematical model was derived which enables the prediction of the free surface velocity for a given rheology of the fluid. The mathematical model was derived from the time-dependent lubrication approximation assuming that the rheology can be described by the Ellis model, a three parameter constitutive equation. From the experimental data and the mathematical model, the optimal rheological parameters to minimise the difference between the model and the experiment were found. The parameter identification method employed was based on a grid search method which involved a se- 

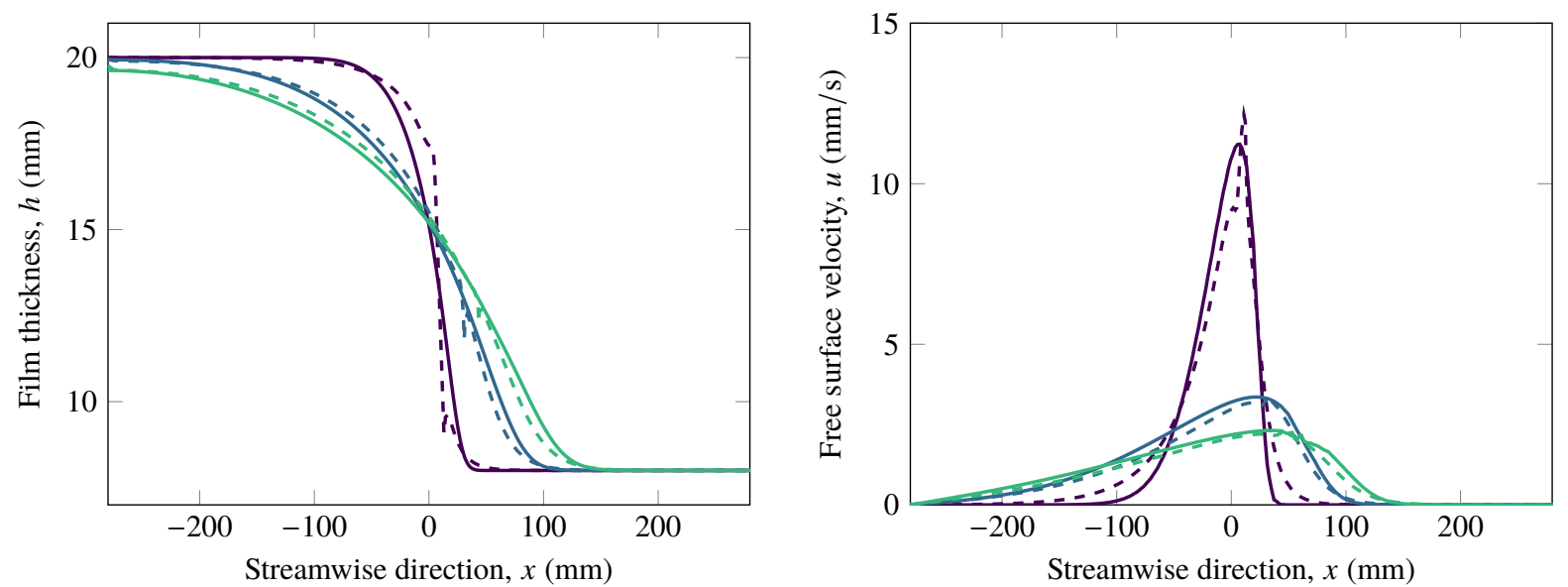

Figure 11: Comparison between Navier-Stokes and the lubrication approximation using the Ellis parameters obtained from the rheometer data in terms of: (a) film thickness, and (b) free surface velocity, at three different time periods for molasses.

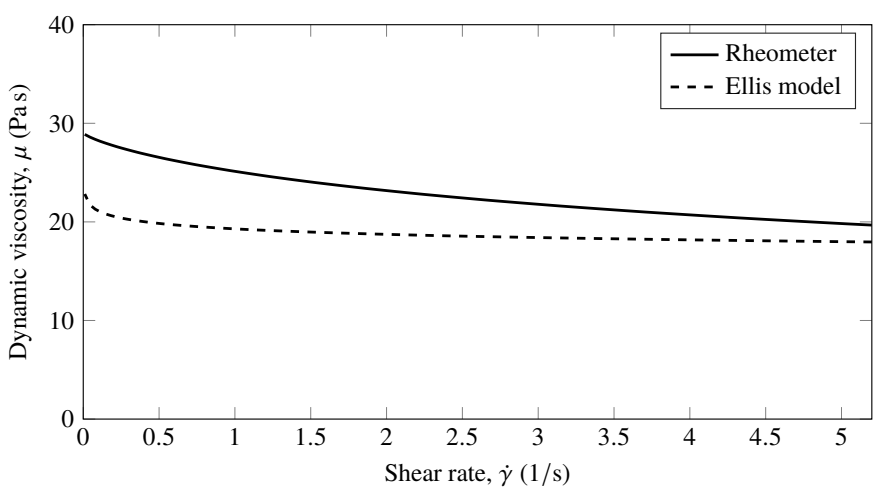

Figure 12: Non-Newtonian viscosity profile against shear rate for molasses, using the rheometer data and the parametric identification data using the Ellis model.

quential parametric study. We identified the rheological parameters for both synthetic and real experimental data, obtained by analysing the dam-break problem using molasses. The results were verified by comparing the experimental free surface velocity data with the computed velocity, calculated according to the ${ }_{450}$ fitted rheological parameters. The compatibility between experimental and simulation was good which indicates that the solution of the parameter identification was valid or a well-posed mathematical problem. A sensitivity analysis was implemented by adding random noise data to the computed free surface ve- 455 locity, and we found that the robustness of the model was excellent up to $40 \%$ noise. Results with molasses showed promise with the maximum difference between the reconstructed and

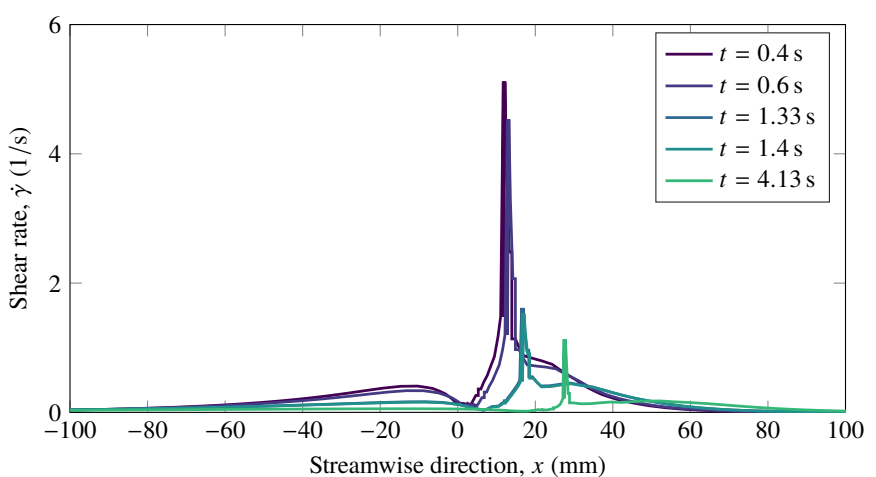

Figure 13: Shear rate of molasses near where the gate was removed.

true flow curves at around $25 \%$. Justification for these differences were provided in the previous section. Notwithstanding the above limitations and given the fact that in some circumstances there are no practical ways to measure the rheology of a fluid, this method could provide a useful alternative.

\section{References}

Afanasiev K, Münch A, Wagner B. Landau-Levich problem for non-Newtonian liquids. Physical Review E 2007;76(3):036307.

Al-Behadili A, Sellier M, Nokes R, Moyers-Gonzalez M, Geoghegan P. Rheometry based on free surface velocity. Inverse Problems in Science and Engineering 2018;Revised - awaiting final decision(-):-.

Berger RE, Corrsin S. A surface tension gradient mechanism for driving the pre-corneal tear film after a blink. Journal of biomechanics 1974;7(3):22538.

Braun RJ, Usha R, McFadden GB, Driscoll TA, Cook LP, King-Smith PE. Thin 
film dynamics on a prolate spheroid with application to the cornea. Journal of Engineering Mathematics 2012;73(1):121-38.

Campagnol J, Radice A, Nokes R, Bulankina V, Lescova A, Ballio F. La-510 grangian analysis of bed-load sediment motion: database contribution. Journal of Hydraulic Research 2013;51(5):589-96.

Charpin JPF, Lombe M, Myers TG. Spin coating of non-Newtonian fluids with a moving front. Physical Review E 2007;76(1):016312.

Eswaran M, Singh A, Saha U. Experimental measurement of the surface veloc-515 ity field in an externally induced sloshing tank. Proceedings of the Institution of Mechanical Engineers, Part M: Journal of Engineering for the Maritime Environment 2011;225(2):133-48.

Heining C, Pollak T, Aksel N. Pattern formation and mixing in threedimensional film flow. Physics of Fluids 2012;24(4):042102.

Hewson RW, Kapur N, Gaskell PH. A model for film-forming with Newtonian and shear-thinning fluids. Journal of Non-Newtonian Fluid Mechanics 2009; $162(1-3): 21-8$

Johnston BM, Johnston PR, Corney S, Kilpatrick D. Non-Newtonian blood flow in human right coronary arteries: steady state simulations. Journal of Biomechanics 2004;37(5):709-20.

Jossic L, Lefevre P, de Loubens C, Magnin A, Corre C. The fluid mechanics of shear-thinning tear substitutes. Journal of Non-Newtonian Fluid Mechanics 2009;161(1-3):1-9.

Kheyfets VO, Kieweg SL. Gravity-driven thin film flow of an Ellis fluid. Journal of Non-Newtonian Fluid Mechanics 2013;202:88-98.

Landel JR, McEvoy H, Dalziel SB. Cleaning of viscous drops on a flat inclined surface using gravity-driven film flows. Food and Bioproducts Processing 2015;93:310-7.

Meselhe E, Peeva T, Muste M. Large scale particle image velocimetry for low velocity and shallow water flows. Journal of Hydraulic Engineering 2004;130(9):937-40.

Miller K, Pike D. Surface active properties of cane molasses. In: Proc. Aust. Soc. Sugar Cane Technol. volume 15; 1993. p. 208-13.

Morris S, Sellier M, Behadili ARA. Comparison of lubrication approximation and navier-stokes solutions for dam-break flows in thin films. arXiv preprint arXiv:170800976 2017;

Myers TG. Application of non-Newtonian models to thin film flow. Physical Review E - Statistical, Nonlinear, and Soft Matter Physics 2005;72(6):066302.

Nokes R. Streams version 2.03: System Theory and Design. Department of Civil and Natural Resources Engineering, University of Canterbury, New Zealand; 2014 .

Roy SC. Withdrawal of cylinders from non-newtonian fluids. The Canadian Journal of Chemical Engineering 1971;49(5):583-9.

Schwartz LW, Eley RR. Flow of architectural coatings on complex surfaces; theory and experiment. Journal of Engineering Mathematics 2002;43(24):153-71.

Sellier M. Inverse problems in free surface flows: a review. Acta Mechanica 2016;227(3):913-35.
Sokoray-Varga B, Józsa J. Particle tracking velocimetry (ptv) and its application to analyse free surface flows in laboratory scale models. Periodica Polytechnica Civil Engineering 2008;52(2):63.

Tallmadge JA. Remarks on the withdrawal problem for ellis fluids. AIChE Journal 1966;12(4):810-2.

Weidner DE, Schwartz LW. Contact-line motion of shear-thinning liquids. Physics of Fluids 1994;6(11):3535-8.

Weitbrecht V, Kühn G, Jirka G. Large scale piv-measurements at the surface of shallow water flows. Flow Measurement and Instrumentation 2002;13(5$6): 237-45$. 